\title{
Depleting endogenous proteins
}

Using antibodies to target specific proteins for rapid degradation is a versatile approach for studying the function of endogenous proteins.

Characterizing the function of a molecule in physiological processes requires the ability to acutely delete it. Many methods can interfere with gene expression at the RNA or DNA level. However, none of these methods directly target proteins, which is problematic for proteins with low turnover.

Melina Schuh from the Max Planck Institute for Biophysical Chemistry in Göttingen, Leo James from MRC Laboratory of Molecular Biology in Cambridge, UK, and their colleagues have developed the aptly named Trim-Away method for targeting proteins for degradation. Their method takes advantage of an immune mechanism mediated by the TRIM 21 receptor. TRIM 21 binds the invariant 'Fc' region of an antibody, while the antibody's variable region binds its spe- cific target. The complex is then degraded by the proteasome. By delivering TRIM21 and an antibody against a protein of interest, the researchers could deplete a protein of interest rapidly over the course of minutes. Schuh, James and colleagues showcased Trim-Away in a variety of cell types. In mammalian oocytes, where transcriptional silencing renders gene-targeting methods inapplicable, their method was particularly useful. They showed that Trim-Away can target proteins in multiple cellular locations. Importantly, Trim-Away could degrade long-lived proteins like Rec8, producing the predicted loss of sister chromatid cohesion. Trim-Away also worked in mammalian cell lines, where the authors could target signal transduction pathways, including some otherwise inaccessible to current inhibitors, and in primary human macrophages. Typical RNA-targeting methods are unsuitable for these cells, since the cells normally activate in response to RNAs.
Crucially, Trim-Away is specific, since phenotypes can be reversed by restoring expression of the target protein. In one tantalizing example, Trim-Away was able to target a disease-specific variant of huntingtin, the protein implicated in Huntington's disease.

Other approaches to directly target proteins employ small peptides or molecules to tag proteins for degradation. However, existing methods require altering the target protein itself, or are restricted by peptide-target affinity and have a limited target set. TrimAway can target, in principle, any protein or protein variant for which there is a specific antibody or nanobody, making this a versatile and potentially broadly applicable tool for direct depletion of endogenous protein.

\section{Ada Yee}

\section{RESEARCH PAPERS}

Clift, D. et al. A method for the acute and rapid degradation of endogenous proteins. Cell 172, 16921706 (2017).

Corrected after print 7 February 2018. 


\section{Erratum: Depleting endogenous proteins}

Ada Yee

Nat. Methods 15, 17 (2018); published online 3 Jan 2018; corrected after print 7 February 2018

In the version of this article initially published, the affiliation of Leo James was incorrectly stated as Cambridge University; the correct affiliation is MRC Laboratory of Molecular Biology, Cambridge. Furthermore, the antibody receptor described was incorrectly called TRIM2; the correct name is TRIM21. The errors have been corrected in the HTML and PDF versions of the article. 